\title{
One year of the pandemic - how European endocrinologists responded to the crisis: a statement from the European Society of Endocrinology
}

\begin{abstract}
A Giustina1, M Marazuela², M Reincke ${ }^{3}$, B O Yildiz ${ }^{4}$ and M Puig-Domingo5
${ }^{1}$ Institute of Endocrine and Metabolic Sciences, Vita-Salute San Raffaele University and IRCCS San Raffaele Hospital, Milano, Italy, ${ }^{2}$ Department of Endocrinology, Hospital Universitario de la Princesa, Instituto de Investigación de la Princesa, Universidad Autónoma de Madrid, Madrid, Spain, ${ }^{3}$ Department of Medicine IV, Faculty of Medicine, University Hospital Munich, LMU, Munich, Germany, ${ }^{4}$ Division of Endocrinology and Metabolism, Department of Internal Medicine, Hacettepe University School of Medicine, Hacettepe Ankara, Turkey, and ${ }^{5}$ Endocrinology and Nutrition Service, Department of Medicine, Germans Trias i Pujol Health Science Research Institute and Hospital, Universitat Autònoma de Barcelona, Badalona, Spain
\end{abstract}

Correspondence should be addressed to A Giustina

Email

giustina.andrea@hsr.it

\begin{abstract}
Changes that COVID-19 induced in endocrine daily practice as well as the role of endocrine and metabolic comorbidities in COVID-19 outcomes were among the striking features of this last year. The aim of this statement is to illustrate the major characteristics of the response of European endocrinologists to the pandemic including the disclosure of the endocrine phenotype of COVID-19 with diabetes, obesity and hypovitaminosis D playing a key role in this clinical setting with its huge implication for the prevention and management of the disease. The role of the European Society of Endocrinology (ESE) as a reference point of the endocrine community during the pandemic will also be highlighted, including the refocusing of its educational and advocacy activities.
\end{abstract}

\section{Introduction}

The Coronavirus disease 2019 (COVID-19) pandemic had, and still is having, a terrible impact on the life of every human being and has also heavily affected the professional life of all health professionals. In particular, young endocrinologists have often been relocated in the first line in the fight against COVID-19 (1) including the tremendous vaccination effort at the population level.

Moreover, many colleagues all over the world contributed to the huge effort of defining the endocrine phenotype of COVID-19 that prompted the understanding of the key role of endocrinologists in the prevention and even management of a disease which apparently was so far from our field of interest and expertise $(2,3)$. In fact,
COVID-19 initially defined as a predominantly respiratory syndrome, caused by severe acute respiratory syndrome coronavirus 2 (SARS-CoV-2), was progressively shown to have multiple systemic extrapulmonary manifestations which increase the lethality of COVID-19 including, besides the endocrine involvement, vascular, cardiac, kidney, gastrointestinal and CNS complications (4) due to the ubiquitous expression of angiotensin-converting enzyme 2 (ACE2), the receptor which is responsible for the cellular entry of SARS-CoV-2 (5), widespread endothelial damage and altered immune response (6).

Furthermore, in order to grant the continuity of endocrine care, many endocrinologists shifted their

Published by Bioscientifica Ltd. 
practice toward a telemedicine approach (7). Finally, the Scientific Societies and the European Society of Endocrinology (ESE), in particular, decided to stand as a reference point for all the members, offering educational tools as well as financial support for specific COVID-19 research to the global endocrine community.

Therefore, due to the relevant involvement and increased responsibility of endocrinologists worldwide in the persisting COVID-19 pandemic, as well as due to the rapidly evolving knowledge on this new disease and its consequences, we have been appointed by the ESE to write a statement on how endocrinologists in Europe, under the ESE umbrella, reacted to all the afore-mentioned aspects of the critical situation created by the COVID-19 pandemic. One important aspect of this statement is that it is entirely based on data coming from the field published in the literature or available through the ESE website.

\section{Relocation of endocrinologists to COVID- 19 patients care}

During the persisting COVID-19 outbreak (we are currently facing the 'third wave' of the pandemic), endocrinologists from all over Europe discovered themselves to be crucial in the fight against the COVID-19. As a matter of fact, they moved first with self-sacrifice and decision to be the first line of care of affected patients, in collaboration with other physicians such as those in internal medicine and emergency units (2). In fact, due to its systemic involvement, internist management of COVID-19 is advised, and many endocrinologists and endocrinology trainees have been called to help in hospitals facing the epidemic crisis (1).

An interesting survey of the directors of more than 30 Italian endocrinology residency programs on the number of hospital beds, physicians, residents, nurses and technicians made available by their schools for Italian health system reorganization was carried out during the first wave of the pandemic (1). In one-quarter of cases, the whole endocrine department was converted to host COVID-19 patients (up to more than one-third of departments in Northern Italy). At least half of the ordinary beds were devoted to COVID19 care in most centers particularly in the north. Overall, less than half of the departments maintained their own endocrine beds.

In about $15 \%$ of endocrine divisions in Northern Italy, the entire staff was dedicated to handling COVID19 patients. However, in two-thirds of sites of north and center Italy, at least half of the specialized personnel was dedicated to the emergency. This means that more than
150 hospitalists/academic endocrinologists (out of a total of about 500) dedicated most of their working hours to COVID-19-related activities (a vast majority of them in northern centers). Similarly, a great part (slightly less than 200) of endocrine nurses dedicated most of their time to COVID-19 healthcare. This reorganization resulted in a reduction or suspension of the endocrine day-hospital admissions and routine visits for the vast majority of sites, while urgent and non-deferrable outpatient care was almost universally preserved (1).

About half of the Italian postgraduate schools, prevalently in the north, made available up to half of their own trainees, which corresponds to slightly less than 200 young in-training endocrinologists who mainly volunteered to work in COVID-19 wards (1). Interestingly, in-training endocrinologists were also recently massively involved in the anti-COVID-19 vaccination of the Italian population.

In a study performed by the Spanish Society of Endocrinology (SEEN) on more than 400 endocrinologists, more than half had worked in the first line in the management of COVID-19 patients, and 10\% had confirmed SARS-CoV-2 infection. Although consultation on endocrinology decreased by $68 \%$, consultation on nutrition increased by $36 \%$ during the pandemic (8). Regarding training in endocrinology, another Spanish survey showed that residents needed to change their training program during the pandemic, losing important rotations that could affect their future professional activity (9).

In summary, these interesting surveys showed that in many parts of Europe, endocrinologists crucially supported their national health systems in the fight against COVID-19 while continuing to grant the best possible management of endocrine patients (10). In fact, the paramount importance of the continuity of endocrine care is underlined by a recent study performed in Northern Italy which showed that during the first lockdown, a significant increase in non-COVID-19 out-of-hospital mortality was mostly due to endocrine, nutritional and metabolic conditions (slightly less than 80\%) (11).

\section{Telemedicine in endocrinology}

This continuity of endocrine care was possible due to the wide use of telemedicine in endocrine centers (7). In a recent survey of more than 100 international pituitary specialists, only less than half reported that the COVID-19 pandemic negatively affected their ability to 
communicate with acromegaly patients (12). Most of the respondents experienced good success with remote patient management, with the highest proportion of those surveyed (55.9\%) indicating that remote methods improved their ability to communicate with their patients. Telephone and email were the most commonly employed means of communication with acromegaly patients, and remote consultations were most commonly used for postdiagnostic discussion of the condition and treatment options whereas were employed to a lesser extent for diagnostic consultations, discussion of alternative treatment pathways and for training of the patient or a family member on acromegaly treatment. Interestingly, more than $40 \%$ of respondents assessed via telemedicine the urgency of a treatment change (12). Moreover, more than two-thirds of respondents indicated that they would continue to use methods of consultation experienced during the COVID-19 pandemic in the presumed postCOVID-19 environment. In fact, they reported virtual visits as a driver of improvements in patient care as they reduce the burden of hospital visits (12).

Furthermore, in another cross-sectional, online survey among 181 US adult endocrinologists, the adoption rate of telehealth practices during the COVID-19 was investigated. Respondents were prevalently young females working in hospitals or academic settings. Almost half of the surveyed endocrinologists switched to completely virtual visits, and an additional relevant percentage switched to a majority of virtual visits, with some in-person outpatient visits. Interestingly, there was a significantly higher adoption rate of telehealth among endocrinologists younger than or equal to 40 years of age (7). Furthermore, in a Spanish study, telehealth endocrine practice increased from $19.5 \%$ to $97.8 \%$ during the pandemic (8).

The importance of the continuity of routine endocrine care during and in spite of the pandemic was not only related to the necessity to avoid a rise in non-COVID-19 morbidity and mortality (11) but also due to the predisposing role of chronic endocrine and metabolic conditions to severe COVID-19 $(2,3)$.

The results of these surveys suggested that endocrine practice can be well suited to provide virtual care to patients with endocrine and metabolic disorders and that the COVID-19 pandemic provided an opportunity to adapt to and adopt a 'new normality' which promises to create a new continuum of care better suited to the presumed postCOVID-19 environment.

On the otherhand, theadaptation tovideoconsultation has also limitations: questionnaire-based surveys showed that patients with osteoporosis were experiencing a drastic decrease in osteoporosis consultations of up to $70 \%$ during the pandemic, without telephone consultations and video consultations being able to compensate for this decrease (13). In addition, reduced-DXA measurements and risk of discontinuation of anti-osteoporotic treatments (14), although they do not appear to predispose to COVID-19 (15), were reported.

The new virtual landscape will clearly still include both usual pre-COVID practices such as face-to-face consultations and also new approaches (such as remote consultations) that may decrease the burden of hospital visits with the ultimate goal of providing the best possible care for endocrine patients. Open issues for routine telemedicine approach implementation are remote consultations reimbursement because they are not yet recognized by most healthcare providers as the in-person patient visits as well as possible legal issues due to risk of data breaching, if not dedicated electronic media are used (12).

\section{Discovery of the endocrine phenotype of COVID-19}

European endocrinologists, besides their efforts in the first line of COVID-19 patient care, greatly contributed to the profiling of an endocrine phenotype of COVID-19 $(2,3,16)$. In fact, they played a key role in the evolving knowledge about a major endocrine and metabolic involvement in COVID-19 with many endocrine organs, tissues and molecules being directly or indirectly affected or negatively influencing outcomes of COVID-19 (2, 3).

A well-defined endocrine phenotype can be of help in preserving the health status and preventing adverse COVID-19 outcomes in both the general population and in people affected by different endocrine diseases. People with diabetes, in particular, are at high risk of severe COVID19 if infected by SARS-Cov-2, which can also directly affect the beta-cell function. Moreover, other endocrine diseases such as obesity, hypovitaminosis D and adrenal dysfunctions may impact on susceptibility and severity of COVID-19 $(2,3)$.

In fact, the most frequent phenotype of a COVID patient requiring hospitalization is an aged male (17) who is bearing cardio-metabolic comorbidities included hypertension (30-55\%), diabetes mellitus (15-33\%) and obesity (20-40\%). Importantly, mortality in either type 1 or type 2 diabetes has consistently increased during the year of pandemic, not only due to co-existing co-morbidities but also due to poor glyco-metabolic control. In fact, the 
last $\mathrm{HbA1c}$ previous to SARS-CoV-2 infection showed a linear relationship to outcomes and particularly to mortality with HbA1c higher than 10\% (18). Furthermore, obesity was consistently shown to amplify the deleterious effects of diabetes upon mortality and severe outcomes (19). One of the emerging features of diabetes in COVID19 which was reported by European endocrinologists from the field was the possible bidirectional relationship between diabetes and COVID-19 (20). In fact, worsening of hyperglycemia in patients with known diabetes, often complicated by ketoacidosis or hyperosmolarity with high-dose insulin requirements and also the new onset of diabetes in COVID-19 patients previously non-diabetic was consistently reported disclosing a potential diabetogenic action of SARS-CoV-2 (20).

Hypocalcemia, hypovitaminosis D and morphometric vertebral fractures were also reported by European endocrinologists as major biochemical and clinical features of COVID-19 being associated with outcomes such as inflammatory parameters, need for hospitalization, mechanical ventilation, ICU admission and mortality $(21,22,23)$. In particular, widespread hypovitaminosis $\mathrm{D}$ in Mediterranean countries was proposed from the beginning of the pandemic as one of the factors in exposing those countries to high risk of COVID-19 and severe diseases (24) since it was reported to predispose to systemic infections impairing immune responses with potential preventive role of vitamin D supplementation toward respiratory infections (25). Poor vitamin D status was also reported in the majority of the studies to predict disease severity, including chest CT stage, and mortality risk being also associated with severe endocrine comorbidities of COVID-19 $(16,26)$. Finally, in a Spanish pilot trial, COVID-19 hospitalized patients with pneumonia randomly given standard care combined with calcifediol showed reduced requirement of ICU admission without deaths (27).

Interestingly, the impact of COVID-19 on the nutritional status of the affected patients was also demonstrated to be a major issue contributing to the dysmetabolic profile of high-risk patients, mostly through the impact of sarcopenia (28) developed by aged patients. A study performed in Spain (29) showed that those patients with more severe COVID-19 and requiring ICU or semicritical vital support presented an intense deterioration of nutritional status with important weight loss and sarcopenia development, thus emphasizing the necessity of specific nutritional support with high protein content diet in all hospitalized cases and artificial nutrition in about $15 \%$ of them.
The results of a small recently published phone interview conducted in Northern Italy reported that around $3 \%$ of patients with Cushing's disease had confirmed COVID-19 vs less than $1 \%$ of the general population during the first wave of the pandemic, and with severe clinical presentation reported particularly in patients with active disease (30) suggesting that patients with chronic hypercortisolism may be at high risk of more severe COVID-19 as also shown in a recently published series from Russia (31).

Specific involvement of the pituitary and thyroid glands in the endocrine phenotype of COVID-19 was recently reported including pituitary apoplexy, hyponatremia and atypical thyroiditis $(32,33)$.

Overall, the contribution of European endocrinologists to the knowledge and definition of the endocrine profile of COVID-19 was of paramount importance prompting the now widely accepted notion of the key role of endocrine abnormalities in the outcome of COVID-19 and the great relevance of endocrine and metabolic health of the population, particularly in the pandemic conditions.

\section{ESE and COVID-19}

ESE activities during this last year were also substantially refocused due to the pandemic in order to better serve as the reference point of the global endocrine community and its membership. In fact, the decision between suspending all educational activities, as meetings in person were no longer possible all over Europe or maintaining them switching to a virtual modality was taken almost immediately after the start of the spreading of the pandemic to Europe. Of particular note, the first webinar of the series ESE Talks was held in mid-May 2020. Clearly, the focus of these webinars was on COVID-19 and related endocrine comorbidities. One of the interesting features of these first webinars was that endocrinologists from almost 100 countries attended the events, suggesting that despite the emergency situation, there was a strong need to communicate among peers and that the virtual modality was able to reach much more people than in in-person meetings, breaking, therefore, the borders and social and economic hurdles that were preventing many endocrinologists to attend usual meetings. This was perfectly fitting with the inclusion plan that was implemented in the last ESE presidential term. Along these lines, also the major educational activity of ESE, that is, the European Congress of Endocrinology (ECE), was transformed into a fully virtual initiative. More than 7000 people following the opening ceremony 
of the meeting was the largest audience ever viewing an ECE session (www.ese-hormones.org).

Besides the virtual educational activities, ESE decided to have an even more active role in the endocrine community through publishing two ESE statements on COVID-19 and endocrine disease, including practical recommendations for clinical endocrinologists (ESE decalogs) $(2,34)$. The first of these statements was downloaded more than 15 000 times and already was cited more than 100 times in international literature, demonstrating to be an essential resource for endocrinologists all over the world. Moreover, the European Journal of Endocrinology, the official journal of ESE, published a series of reviews on endocrine conditions in the COVID-19 era to which many endocrinologists from all over Europe and beyond contributed significantly (35).

ESE also issued a call for research grants for the study of endocrine conditions in COVID-19, which was repeated after several months due to the many interesting projects which were presented (www.ese-hormones.org).

Moreover, during the COVID-19 pandemic an intense activity was undertaken at the institutional level within the European Parliament and the European Commission. The creation of the European Hormone and Metabolism Foundation (ESE Foundation), and the production of the first ESE white paper on endocrinology entitled 'Hormones in European Health Policies: How endocrinologists can contribute to a healthier Europe,' being huge steps toward a strong positioning of endocrinology in the European health authorities agenda. The collaboration with the Endocrine Society and most of the other important international endocrine societies allowed an effective and coordinated global response to the COVID-19 pandemic demonstrating a strong global endocrine community according to our inclusion plan (www.ese-hormones.org).

Another area heavily impacted by COVID-19, according to a study by EURORDIS, was severe impairment of care for $83 \%$ of people living with a rare disease (36), which often fall in the endocrine category. As a commitment to rare endocrine diseases, ESE established in 2019 an Endocrine Rare Disease Committee, which started, in collaboration with the Endocrine European Reference Network (ENDO-ERN), an initiative to collect essential data concerning specific groups of patients with rare endocrine conditions, who were also affected by COVID-19, to objectively document rare endocrine patient care during the pandemic (37).

Finally, due to the questioning of the safety and opportunity of COVID-19 vaccination in some patients with endocrine diseases, such as autoimmune thyroiditis, ESE recently released an ad hoc statement (38). In fact, patients with stable diabetes and obesity were enrolled in vaccine trials submitted to FDA and EMA, and similar efficacy and safety were reported in those patients as compared to healthy subjects (39). Consequently, the ESE statement supported the recommendation that COVID-19 vaccination should not be handled differently in patients with stable endocrine diseases such as autoimmune thyroiditis, Grave's disease, Addison's disease, pituitary adenomas, diabetes type 1 and 2 and obesity as compared to the general population. The ESE statement recommended informing patients with adrenal insufficiency of how to handle possible vaccine side effects such as fever (38).

\section{Conclusions}

European endocrinologists during the COVID-19 pandemic were key in the response to the emergency which also once again confirmed the paramount importance of the preventive work they do in controlling diabetes, obesity and osteoporosis/hypovitaminosis D as well as rarer endocrine diseases which contribute to what we already can define the endocrine phenotype of the disease. The use of telemedicine which demonstrated to be an extraordinary resource to grant the continuity of endocrine care will likely change the endocrine practice forever. ESE stood as a strong reference point for all endocrinologists in Europe and beyond, continuing all educational activities and significantly contributing to the international literature and to the health authorities in alliance with other scientific bodies and societies sharing the same values and vision through its inclusive philosophy within an endocrine community which understood that only united and truly global action will make it possible to deal with the challenges of the pandemic that we will face in the future.

\section{Declaration of interest}

The authors declare that there is no conflict of interest that could be perceived as prejudicing the impartiality of this commentary.

\section{Funding}

This research did not receive any specific grant from any funding agency in the public, commercial or not-for-profit sector.

\section{Ethics}

Due to the nature of the article (review) no study-specific approval by the appropriate ethics committee for research involving humans and/ or animals, neither informed consent if the research involved human 
participants, and a statement on welfare of animals if the research involved animals is provided.

\section{References}

1 Isidori AM, Jannini EA, Lenzi A, Ghigo E \& Board of Full Professors in Endocrinology, Directors of the Endocrinology, Metabolic Disorders Residency Program. The Italian endo-COVID-19. Our response to the emergency. Journal of Endocrinological Investigation 202043 1039-1041. (https://doi.org/10.1007/s40618-020-01279-5)

2 Puig-Domingo M, Marazuela M \& Giustina A. COVID-19 and endocrine diseases. A statement from the European Society of Endocrinology. Endocrine 202068 2-5. (https://doi.org/10.1007/ s12020-020-02294-5)

3 Marazuela M, Giustina A \& Puig-Domingo M. Endocrine and metabolic aspects of the COVID-19 pandemic. Reviews in Endocrine and Metabolic Disorders 202021 495-507. (https://doi.org/10.1007/s11154020-09569-2)

4 Gupta A, Madhavan MV, Sehgal K, Nair N, Mahajan S, Sehrawat TS, Bikdeli B, Ahluwalia N, Ausiello JC, Wan EY et al. Extrapulmonary manifestations of COVID-19. Nature Medicine 202026 1017-1032. (https://doi.org/10.1038/s41591-020-0968-3)

5 Lan J, Ge J, Yu J, Shan S, Zhou H, Fan S, Zhang Q, Shi X, Wang Q, Zhang L et al. Structure of the SARS-CoV-2 spike receptor-binding domain bound to the ACE2 receptor. Nature 2020581 215-220. (https://doi.org/10.1038/s41586-020-2180-5)

6 Ackermann M, Verleden SE, Kuehnel M, Haverich A, Welte T, Laenger F, Vanstapel A, Werlein C, Stark H, Tzankov A et al. Pulmonary vascular endothelialitis, thrombosis, and angiogenesis in Covid-19. New England Journal of Medicine 2020383 120-128. (https://doi. org/10.1056/NEJMoa2015432)

7 Madduri S, Chowdhary R \& Sethu Reddy S. Telehealth adoption among endocrinologists during the covid-19 pandemic. Endocrine Practice 202026 846-856. (https://doi.org/10.4158/EP-2020-0237)

8 Breton-lesmes I, Zugasti- Murillo A \& Rubio-Herrera MA. Como ha afectado la pandemia covid-19 en la actividad de los socios de la Seen? Endocrinología, Diabetes y Nutrición 2020671.

9 Zugasti A, Ballesteros-Pomar M, Breton-lesmes I, Escalada J, RubioHerrera MA, Tejera C, Pines-Corrales P \& Hanzu F. How has the covid-19 pandemic affected training in endocrinology and nutrition. Endocrine Abstracts 202173 AEP837. (https://doi.org/10.1530/ endoabs.70.AEP837)

10 Kaiser UB, Mirmira RG \& Stewart PM. Our response to COVID-19 as endocrinologists and diabetologists. Journal of Clinical Endocrinology and Metabolism 2020105 dgaa148. (https://doi.org/10.1210/clinem/dgaa148)

11 Santi L, Golinelli D, Tampieri A, Farina G, Greco M, Rosa S, Beleffi M, Biavati B, Campinoti F, Guerrini S et al. Non-COVID-19 patients in times of pandemic: emergency department visits, hospitalizations and cause-specific mortality in Northern Italy. PLoS ONE 202116 e0248995. (https://doi.org/10.1371/journal.pone.0248995)

12 Giustina A, Legg E, Cesana BM, Frara S, Mortini P \& Fleseriu M. Results from ACROCOVID: an international survey on the care of acromegaly during the COVID-19 era. Endocrine 202171 273-280. (https://doi. org/10.1007/s12020-020-02565-1)

13 Singer AJ, Fuggle NR, Gill CB, Patel AR, Medeiros AP \& Greenspan SL. COVID-19 and effects on osteoporosis management: the patient perspective from a National Osteoporosis Foundation Survey. Osteoporosis International 202132 1-4. (https://doi.org/10.1007/s00198021-05836-3)

14 Fuggle NR, Singer A, Gill C, Patel A, Medeiros A, Mlotek AS, Pierroz DD, Halbout P, Harvey NC, Reginster JY et al. How has COVID-19 affected the treatment of osteoporosis? An IOF-NOF-ESCEO Global Survey. Osteoporosis International 202132 611-617. (https://doi.org/10.1007/ s00198-020-05793-3)
15 Formenti AM, Pedone E, di Filippo L, Ulivieri FM \& Giustina A. Are women with osteoporosis treated with denosumab at risk of severe COVID-19? Endocrine 202070 203-205. (https://doi.org/10.1007/ s12020-020-02500-4)

16 Giustina A. Hypovitaminosis D and the endocrine phenotype of COVID-19. Endocrine 202172 1-11. (https://doi.org/10.1007/s12020021-02671-8)

17 Brandi ML \& Giustina A. Sexual dimorphism of coronavirus 19 morbidity and lethality. Trends in Endocrinology and Metabolism 2020 31 918-927. (https://doi.org/10.1016/j.tem.2020.09.003)

18 Cariou B, Hadjadj S, Wargny M,Pichelin M, Al-Salameh A, Allix I, Amadou C, Arnault G, Baudoux F, Bauduceau B et al. Phenotypic characteristics and prognosis of inpatients with COVID-19 and diabetes: the CORONADO study. Diabetologia 202063 1500-1515. (https://doi.org/10.1007/s00125-020-05180-x)

19 Helvaci N, Eyupoglu ND, Karabulut E \& Yildiz BO. Prevalence of obesity and its impact on outcome in patients with COVID-19: a systematic review and meta-analysis. Frontiers in Endocrinology 202112 598249. (https://doi.org/10.3389/fendo.2021.598249)

20 Rubino F, Amiel SA, Zimmet P, Alberti G, Bornstein S, Eckel RH, Mingrone G, Boehm B, Cooper ME, Chai Z et al. New-onset diabetes in Covid-19. New England Journal of Medicine 2020383 789-790. (https:// doi.org/10.1056/NEJMc2018688)

21 Di Filippo L, Formenti AM, Rovere-Querini P, Carlucci M, Conte C, Ciceri F, Zangrillo A \& Giustina A. Hypocalcemia is highly prevalent and predicts hospitalization in patients with COVID-19. Endocrine 202068 475-478. (https://doi.org/10.1007/s12020-020-02383-5)

22 Hutchings N, Babalyan V, Baghdasaryan S, Qefoyan M, Sargsyants N, Aghajanova E, Martirosyan A, Harutyunyan R, Lesnyak O, Formenti AM et al. Patients hospitalized with COVID-19 have low levels of 25-hydroxyvitamin D. Endocrine 202171 267-269. (https:// doi.org/10.1007/s12020-020-02597-7)

23 di Filippo L, Formenti AM, Doga M, Pedone E, Rovere-Querini P \& Giustina A. Radiological thoracic vertebral fractures are highly prevalent in COVID-19 and predict disease outcomes. Journal of Clinical Endocrinology and Metabolism 2020106 dgaa738. (https://doi. org/10.1210/clinem/dgaa738)

24 Giustina A \& Formenti AM. Preventing a covid-19 pandemic can high prevalence of severe hypovitaminosis D play a role in the high impact of Covid infection in Italy? BMJ $2020368 \mathrm{~m} 810$.

25 Bouillon R, Marcocci C, Carmeliet G, Bikle D, White JH, DawsonHughes B, Lips P, Munns CF, Lazaretti-Castro M, Giustina A et al. Skeletal and extraskeletal actions of vitamin D: current evidence and outstanding questions. Endocrine Reviews 201940 1109-1151. (https:// doi.org/10.1210/er.2018-00126)

26 Bilezikian JP, Bikle D, Hewison M, Lazaretti-Castro M, Formenti AM, Gupta A, Madhavan MV, Nair N, Babalyan V, Hutchings N et al. Mechanisms in endocrinology: vitamin D and COVID-19. European Journal of Endocrinology 2020183 R133-R147. (https://doi.org/10.1530/ EJE-20-0665)

27 Entrenas Castillo M, Entrenas Costa LM, Vaquero Barrios JM, Alcalá Díaz JF, López Miranda J, Bouillon R \& Quesada Gomez JM. Effect of calcifediol treatment and best available therapy versus best available therapy on intensive care unit admission and mortality among patients hospitalized for COVID-19: a pilot randomized clinical study. Journal of Steroid Biochemistry and Molecular Biology 2020203105751. (https://doi.org/10.1016/j.jsbmb.2020.105751)

28 Donini LM, Busetto L, Bauer JM, Bischoff S, Boirie Y, Cederholm T, Cruz-Jentoft AJ, Dicker D, Frühbeck G, Giustina A et al. Critical appraisal of definitions and diagnostic criteria for sarcopenic obesity based on a systematic review. Clinical Nutrition 202039 2368-2388. (https://doi.org/10.1016/j.clnu.2019.11.024)

29 Ramos A, Joaquín C, Ros M, Martín M, Cachero M, Sospedra M, Martínez E, Sánchez-Migallón JM, Sendrós MJ, Soldevila B, , \& PuigDomingo M. Impact of COVID-19 on nutritional status during the first 
wave of the pandemic . Clinical Nutrition 2021 In press. (https://doi. org/10.1016/j.clnu.2021.05.001)

30 Serban AL, Ferrante E, Carosi G, Indirli R, Arosio M \& Mantovani G. COVID-19 in Cushing disease: experience of a single tertiary centre in Lombardy. Journal of Endocrinological Investigation 2021 44 1-2. (https://doi.org/10.1007/s40618-020-01419-x)

31 Belaya Z, Golounina O, Melnichenko G, Tarbaeva N, Pashkova E, Gorokhov M, Kalashnikov V, Dzeranova L, Fadeev V, Volchkov P et al. Clinical course and outcome of patients with ACTH-dependent Cushing's syndrome infected with novel coronavirus disease-19 (COVID-19): case presentations. Endocrine 202172 12-19. (https://doi. org/10.1007/s12020-021-02674-5)

32 Frara S, Allora A, Castellino L, di Filippo L, Loli P \& Giustina A. Covid-19 and the pituitary. Pituitary 202124 465-481. (https://doi. org/10.1007/s11102-021-01148-1)

33 Muller I, Cannavaro D, Dazzi D, Covelli D, Mantovani G, Muscatello A, Ferrante E, Orsi E, Resi V, Longari V et al. SARS-CoV-2-related atypical thyroiditis. Lancet: Diabetes and Endocrinology 20208 739-741. (https:// doi.org/10.1016/S2213-8587(20)30266-7)

34 Puig-Domingo M, Marazuela M, Yildiz B \& Giustina A. COVID-19 and endocrine and metabolic diseases. An updated statement from the European Society of Endocrinology. Endocrine 202172 301- 316 (https://doi.org/10.1007/s12020-021-02734-w)
35 Newell-Price J, Nieman LK, Reincke M \& Tabarin A. Endocrinology in the time of COVID-19: management of Cushing's syndrome. European Journal of Endocrinology 2020183 G1-G7. (https://doi.org/10.1530/EJE-20-0352)

36 EURORDIS How has Covid-19 impacted people with rare disease. Rare barometer - A Eurordis Initiative 2020. (http://download2.eurordis. org/rbv/covid19survey/covid_infographics_final.pdf)

37 Reincke M \& Hokken-Koelega A. Perspectives of the European Society of Endocrinology (ESE) and the European Society of Paediatric Endocrinology (ESPE) on rare endocrine disease. Endocrine 202171 539-541. (https://doi.org/10.1007/s12020-021-02652-x)

38 Luger A, Giustina A \& Peeters R. European Society of Endocrinology (ESE)'s statement concerning COVID19 vaccination: 'follow the same recommendations for patients with stable endocrine disorders as for the general population. 2021European Society of Endocrinology. (available at: https://www.ese-hormones.org/news/ese-news/ european-society-of-endocrinology-ese-s-statement-concerningcovid-19-vaccination-follow-the-same-recommendations-for-patientswith-stable-endocrine-disorders-as-for-the-general-population/)

39 Polack FP, Thomas SJ, Kitchin N, Absalon J, Gurtman A, Lockhart S, Perez JL, Perez Marc G, Moreira ED, Zerbini C et al. Safety and efficacy of the BNT162b2 mRNA Covid-19 vaccine. New England Journal of Medicine 2020383 2603-2615. (https://doi.org/10.1056/ NEJMoa2034577)

Received 17 April 2021

Accepted 14 June 2021 